\title{
CHARACTERISTICS OF BIPOLAR DISORDER PATIENTS WITH MIXED FEATURES
}

\author{
R. Felhi1, A. Bouallagui1, M. Mlika1, O. Moula1, R. Ghachem1 \\ 1Razi Hospital, Psychiatry B, Tunis, Tunisia
}

\section{Introduction:}

The DSM-5 mixed features (Mx) specifier for mood disorders encouraged renewed interest iAn mixed states and led us to pool research findings regarding the characteristics of these episodes. Bipolar patients developing mood episodes with mixed features seem to have similarities, creating a particular "subtype" $(1,2)$.

\section{Objectives:}

The aim of this study is to determine the characteristics of the patients who has developed a depressive or maniac episode with mixed features (Mx).

\section{Methods:}

We determined the sociodemographic and clinical characteristics in 50 outpatient subjects with DSM-5 bipolar disorder type 1 who had affective episodes with mixed features.

\section{Results:}

Mx subjects were mostly female (58,30\%). Half of them had a deceased parent and 9,8\% had an early parental separation. In $58,9 \%$ of cases, the studied population was married with few children $(1,42)$. A family history of bipolar disorder was found in $25 \%$ of patients and none had a family history of suicide attempts. Substance use was found in $12,5 \%$ of cases. Twenty percent of the patients had history of suicide attempts. The average age of onset was 26.33 years and the average age at first admission was 31.88 years. Total duration of major affective illness was 10,42 years. The polarity index was manic in more than half of the patients $(54,2 \%)$.

The average number of hospitalizations was 3.42. Recurrent mixed episodes were found in $16,7 \%$ of cases. The majority of patients were treated with mood stabilizer and neuroleptic combination $(87,5 \%)$ of which the most prescribed was olanzapine and sodium valproate association.

\section{Discussion:}

This study assessed multiple, clinically relevant characteristics of bipolar disorder type I patients with mixed features, meeting diagnostic criteria proposed by the DSM5. Our study showed that the presence of mixed features was more associated with mania $(54,2 \%)$ then with depression $(45,8 \%)$. These results are different from those found in a similar study that was conducted in the USA (3). These findings also highlighted the fact that mixed features were associated with substance abuse which may facilitate an early onset of the illness and result in important suicidal behaviour $(3,4)$. The tendency for episodes with mixed features to recur $(16,7 \%)$ suggests that such states are not random or sporadic. These features, along with an excess of bipolar disorder in first-degree relatives (25\%), support the proposal that mood disorders marked by mixed features may represent a clinically significant, syndromal subtype of mood disorder (3). Clinical significance of identifying such a subtype, based on the present findings taken together, includes its association with severe and possibly poor treatment-responsive illness and with suicidal behaviour. This explains the important use of treatment association $(87,5 \%)$ of antipsychotic and mood-stabilizers and the absence of antidepressant utilization.

\section{Conclusion:}

This study showed that Mx subjects had severe illness with high risk of substance abuse, suicidal behaviour.

Recurrences of episodes with mixed features and poor response to treatments were noted. Thereby, the presence of mixed features modifies the course of the illness and the prognosis which requires increased precaution when dealing with Mx subjects.

2. Muneer A. Staging models in bipolar disorder: a systematic review of the literature. Clin Psychopharmacol Neurosci 2016; 14:117-130.

3. Tondo L, Vazquez GH, Pinna M, Vaccotto PA, Baldessarini RJ. Characteristics of depressive and : bipolar disorder patients with mixed features. Acta Psychiatr Scand 2018:1-10.

4. PERUGI G, ANGST J, AZORIN J-M et al. Mixed features in patients with a major depressive episode: the BRIDGE-II- MIX study. J Clin Psychiatry 2015;76:e351-e358.

5. PERSONS JE, CORYELL WH, SOLOMON DA, KELLER MB, ENDICOTT J, FIEDOROWICZ JG. Mixed state and suicide: is the effect of mixed : suicidal behavior more than the sum of its parts? Bipolar Disord 2018;20:35-41. 